\title{
Charta für den Umgang mit Zuwendungen in Ärztenetzwerken
}

\author{
Im Auftrag des Dachverbandes med-swiss.net*
}

\begin{abstract}
Ärztenetze setzen sich in Managed-Care-Modellen für eine Optimierung von Qualität und Wirtschaftlichkeit der medizinischen Behandlung ein. Sie sollen den unternehmerischen Freiraum mit gemeinsamem Einkauf von Gütern und Leistungen zur Kosteneffizienzsteigerung nutzen können. Mit dieser Charta gibt med-swiss.net als Dachverband der Schweizer Ärztenetzwerke eine Orientierungshilfe, wie mit Rabatten und Sponsoringbeiträgen umzugehen ist. Diese ausgehandelten Zuwendungen sind entweder den Patienten/Versicherungen weiterzugeben oder auf der Ebene der Ärztenetze für patienten- oder netzwerkbezogene Projekte und Aktivitäten einzusetzen. Sie dürfen jedoch nicht in die Tasche des einzelnen Arztes fliessen. Die Ärztenetze geben der Öffentlichkeit Auskunft über die Herkunft und die Verwendung der Zuwendungen.
\end{abstract}

\footnotetext{
* Ulrich Bodmer, Vorstand medswiss.net; Max-Albrecht Fischer, Präsident med-swiss.net; Felix Huber, Leiter MediX Ärzteverbund Zürich; Marcel Marolf, Novartis Pharma Schweiz AG; Christian Marti, Medizinischer Leiter Wintimed; Karl Metzger, Leiter Managed Care BlueCare; Michael Peltenburg, Medizinischer Leiter zmed.

Juristische Beratung: Ursula Eggenberger Stöckli, Fürsprecherin und Apothekerin, Bratschi Emch Rechtsanwälte, Bern und Zürich.
}

Korrespondenz:

Dr. med. Michael Peltenburg 8340 Hinwil

E-Mail: Michael.Peltenburg@hin.ch nachzukommen, ihr Handeln eigenverantwortlich darauf auszurichten und die Charta bei allen Partnern bekanntzumachen.

\section{Grundlagen: Managed Care und Ärztenetzwerke}

Gesundheitssysteme sind im Wandel, was sowohl Chancen als auch Risiken mit sich bringt. Managed Care steht für den Wandel hin zu neuen Versorgungssystemen, hat aber kein einheitliches Erscheinungsbild.

Bei Managed Care geht es um das Management des Versicherten und/oder Patienten und darum, auf das Gesundheitsverhalten und/oder den Therapieplan Einfluss zu nehmen. Managed Care umfasst sowohl die Leistungserbringung als auch die Leistungsfinanzierung und Leistungsbeanspruchung. Leistungserbringer, die sich in Managed-Care-Strukturen begeben, übernehmen zusätzliche Verantwortung. Sie benötigen Freiräume für neue Behandlungs-, Management- und Versicherungsformen und tragen dafür auch ein unternehmerisches Risiko. Damit diese Risiken eingegangen werden, braucht es nicht nur unternehmerischen Freiraum, sondern auch gesetzliche Grundlagen, welche sicherstellen, dass sich das Risiko lohnt und auszahlt. Diese gesetzlichen Grundlagen fehlen zurzeit noch weitgehend [2].

In Managed-Care-Modellen kommen zu den traditionellen Einzelleistungstarifen neue Vergütungsformen. Die Leistungserbringer erhalten eine wirtschaftliche Mitverantwortung (Budgetmitverantwortung), indem ein bestimmter Teil des Kostenrisikos von den Versicherern auf die Ärzte übertragen wird. Diese Verschiebung führt dazu, dass Ärzte in einem Managed-Care-Modell dann wirtschaftlich erfolgreich sind, wenn sie ihre Leistungen möglichst kosteneffizient erbringen. Ärzte in einem Managed-Care-Modell profitieren deshalb von ausgehandelten Vergünstigungen, da diese zu tieferen Behandlungskosten und damit zu einem besseren Betriebsergebnis des entsprechenden Ärztenetzwerkes führen. 
Anders als bei traditionellen Versorgungssystemen kommen Rabatte und andere Zuwendungen bei Managed-Care-Modellen nicht dem einzelnen Arzt persönlich zugute, sondern dem Modell. Sie dienen vor allem der Finanzierung von Projekten oder - in Form von tieferen Prämien - als Wettbewerbsinstrument gegenüber anderen Versorgungssystemen und können auch zur Verbesserung des Betriebsergebnisses beitragen [3].

Diese Charta bezieht sich auf ManagedCare-Modelle, welche auf seiten der Leistungserbringer von Ärztenetzwerken betrieben werden. Ärztenetzwerke im Sinne dieser Charta sind Organisationen, die von eigenständigen Leistungserbringern gebildet werden und der Gesundheitsversorgung dienen. Durch verbindliches Zusammenwirken unter sich, mit netzfremden Leistungserbringern und mit den Kostenträgern, erbringen sie auf die Bedürfnisse der Patientinnen und Patienten ausgerichtete Gesundheitsleistungen. Dieses Zusammenwirken beruht auf vereinbarten Behandlungsprozessen und einer gemeinsamen Betreuungskultur sowie unternehmerischen Organisationsstrukturen. Ärztenetzwerke gehören in der Regel ganz oder teilweise den beteiligten Ärzten und sind meist als juristische Person konstituiert [4].

Ärztenetzwerke sind Versorgungsformen, welche Dienstleistungen für Netzärzte erbringen wie

- den Ausbau und die Verbesserung der Zusammenarbeit zwischen Patient, Hausarzt, Spezialarzt, Spital und weiteren Leistungserbringern;

- die aktive Mitgestaltung der medizinischen Versorgung durch die Ärzteschaft;

- die Optimierung des Arzneimitteleinsatzes;

- die Einführung von Steuerungsinstrumenten zur Verbesserung des Kosten-Nutzen-Verhältnisses und der Behandlungsprozesse;

- die Verbesserung des Informationsaustausches und der elektronischen Vernetzung und

- den Einkauf von Arzneimitteln, Medizinprodukten und weiteren Dienstleistungen für die angeschlossenen Netzärzte [4].

Ärztenetzwerke haben bisher keine Behandlungsfunktionen übernommen und gelten deshalb nicht als Leistungserbringer im Sinne des Krankenversicherungsgesetzes (KVG); es ist jedoch denkbar, dass sie in Zukunft zum Beispiel eine Telefontriage durchführen, dem Netzarzt sozialmedizinische Koordinationsaufgaben abnehmen oder Case Managements anbieten. Da die bestehenden Ärztenetzwerke zurzeit noch keine Personen beschäftigen, die Arzneimittel verschreiben oder abgeben, gelten sie nicht als Organisationen im Sinne von Art. 33, Abs. 1 und 2, des Heilmittelgesetzes (HMG). Sie dürfen somit Zuwendungen annehmen. Falls Ärztenetzwerke jedoch selbst Zuwendungen an die Netzärzte gewähren, sind sie Vorteilsgeber gemäss Art. 33 HMG und haben die entsprechenden Vorschriften zu beachten. Zusätzlich sind kantonale Regelungen über die Zusammenarbeit zwischen verschiedenen Medizinalpersonen oder von Medizinalpersonen mit Dritten zu beachten, soweit diese zur Anwendung kommen.

Ärztenetzwerke haben die Zusammenarbeit mit der Industrie, den Versicherungen und den Behörden angeregt, entwickelt und tragen sie mit. Sie stellen die Behandlungsqualität und die Betreuung der Patienten sicher, tragen gleichzeitig Budgetmitverantwortung und erhöhen die Wirtschaftlichkeit, indem sie unter anderem die Menge der erbrachten Leistungen steuern.

Ärztenetzwerke setzen sich bei hoher Versorgungsqualität einen haushälterischen Umgang mit den vorhandenen Mitteln zum Ziel. Sie finanzieren sich vor allem durch Mitgliederbeiträge und Steuerungspauschalen (Vereinbarungen in den Verträgen mit den Versicherern) sowie Sponsoringbeiträge von Dritten. Zusätzlich ermöglicht der gemeinsame Einkauf von Leistungen das Aushandeln von Rabatten. Sponsoringbeiträge und Rabatte werden eingesetzt zur Erhöhung der Effizienz sowie für laufende und neue patienten- und netzwerkbezogene Projekte und Aktivitäten. Dank der Zuwendungen können beispielsweise Patienten mittels Broschüren, Gesundheitsdossiers und Merkblättern informiert und geschult oder Impfkampagnen und Präventivprogramme angeboten werden. Im Ärztenetzwerk werden Zuwendungen verwendet, um neue Modelle und Steuerungsinstrumente zu entwickeln.

Um Transparenz und Vertrauen zu schaffen, geben Ärztenetzwerke Auskunft über die Vereinbarungen zwischen den Partnern und die Herkunft sowie Verwendung der Mittel. Sie gewähren der Öffentlichkeit Einblick in ihre Tätigkeit und beweisen, dass die Verantwortung für die Patienten an erster Stelle steht und trotz finanzieller Unterstützung durch Dritte keine falschen Anreize oder Abhängigkeiten entstehen.

Um ihre hohen Ansprüche zu erfüllen und gegenüber der Öffentlichkeit Transparenz zu schaffen, befolgen Ärztenetzwerke und die ihnen angeschlossenen Netzärzte die folgenden Grundsätze: 


\section{Grundsätze}

1. Ärztenetzwerke geben gegenüber der Öffentlichkeit Auskunft über die Herkunft und die Verwendung von Zuwendungen wie Rabatte und Sponsoringbeiträge.

2. Ärztenetzwerke setzen Zuwendungen zweckbestimmt für patienten- oder netzwerkbezogene Projekte und Aktivitäten ein und stellen sicher, dass das Verschreibungs- oder Abgabeverhalten der Netzärzte durch die Zuwendungen nicht beeinflusst wird. Werden $\mathrm{Zu}$ wendungen an die Netzärzte weitergegeben, sind sie in den Patientenrechnungen oder der Tarifgestaltung mit den Krankenversicherern zu berücksichtigen.

3. Ärztenetzwerke verpflichten sich, für das Verschreiben oder Abgeben von Arzneimitteln keine geldwerten Vorteile anzunehmen oder zu gewähren.

\section{Literatur}

1 Dem med-swiss.net sind zurzeit 25 Ärztenetzwerke bzw. 1318 Netzärzte angeschlossen. Mitgliederverzeichnis siehe www.medswiss.net.ch.

2 Britt F. Managed Care sinnvoll fördern. Managed Care 2004;4:6.

3 Tagmann C. Anreize selbstdispensierender Ärzte, Vergünstigungen auszuhandeln - oder:

Was Managed Care bewirken kann. Managed Care 2004;4:22.

4 In Anlehnung an die Definition des med-swiss.net im Grundlagenpapier «Netzwerke - von der Vision zur Realität», verabschiedet an der Generalversammlung vom 19. Januar 2005.

Die Hinweise für das praktische Vorgehen und gesetzliche Grundlagen finden Sie als pdf-Dokument unter www.saez.ch/charta_netzwerke.pdf 


\title{
Charta für den Umgang mit Zuwendungen in Ärztenetzwerken
}

\author{
Anhang: Hinweise für das praktische Vorgehen und gesetzliche Grundlagen
}

Im Auftrag des Dachverbandes med-swiss.net*

\footnotetext{
* Ulrich Bodmer, Vorstand medswiss.net; Max-Albrecht Fischer, Präsident med-swiss.net; Felix Huber, Leiter MediX Ärzteverbund Zürich; Marcel Marolf, Novartis Pharma Schweiz AG; Christian Marti, Medizinischer Leiter Wintimed; Karl Metzger, Leiter Managed Care BlueCare; Michael keine Rabatte im gesetzlichen Sinn darstellen oder über den zulässigen Rabattbetrag hinausgehen, sind nicht zulässig, falls das Verschreibungs- oder Abgabeverhalten der Netzärzte dadurch beeinflusst wird. Dasselbe gilt für finanzielle Beiträge ohne Zweckbestimmung.
} Peltenburg, Medizinischer Leiter zmed.

Juristische Beratung: Ursula Eggenberger Stöckli, Fürsprecherin und Apothekerin, Bratschi Emch Rechtsanwälte, Bern und Zürich.

\subsection{Umgang mit Rabatten und Sponsoring- beiträgen}

Art. 56, Abs. 3, KVG verlangt, dass Vergünstigungen, die ein Leistungserbringer für die Lieferung
Die nachfolgenden Ausführungen stützen sich auf den Stand des Wissens im Zeitpunkt des Erlasses der Charta. Sie sind regelmässig zu überprüfen und anzupassen, wenn sich neue Erkenntnisse ergeben.

\section{Hinweise für das praktische Vorgehen}

\subsection{Arten von Zuwendungen}

Die Charta gilt für Zuwendungen an Ärztenetzwerke in Form von Rabatten und Sponsoringbeiträgen. Für die Charta gelten die folgenden Umschreibungen (vgl. auch Ziffer 2.3):

- Handelsübliche Rabatte sind Rabatte, die ein Lieferant dem Käufer üblicherweise und ohne spezielle Gegenleistung gewährt.

- Betriebswirtschaftlich gerechtfertigte Rabatte sind Rabatte, die ein Lieferant einem Käufer gewährt als Entschädigung für eine handelsbezogene Leistung oder für Einsparungen im Zusammenhang mit Warenlieferungen wie zum Beispiel die Übernahme von Lagerhaltung und das Einsparen von Transportkosten beim Kauf einer grossen Menge, als Skonto bei sofortiger Bezahlung usw.

- Sponsoringbeiträge sind Zuwendungen, welche für einen bestimmten Zweck, insbesondere für patienten- oder netzwerkbezogene Projekte und Aktivitäten, gewährt werden.

- Finanzielle Beiträge, welche für den Einkauf von Arzneimitteln gewährt werden und von Arzneimitteln oder Medizinprodukten erhält, dem Patienten oder seiner Krankenversicherung weitergegeben werden. Ärztenetzwerke, die nicht direkt gegenüber dem Patienten tätig sind, sind keine Leistungserbringer im Sinne des KVG und deshalb von dieser Weitergabepflicht grundsätzlich nicht betroffen.

Art. 33 HMG verbietet das Versprechen oder Gewähren bzw. das Annehmen oder Fordern von geldwerten Vorteilen an bzw. durch Organisationen, die Personen beschäftigen, welche Arzneimittel verschreiben oder abgeben. Die bestehenden Ärztenetzwerke beschäftigen zurzeit noch keine solchen Personen und Art. 33 HMG ist nicht anwendbar, soweit das Ärztenetzwerk Zuwendungen erhält und diese patientenbezogen oder für Projekte im Ärztenetzwerk einsetzt. Falls das Ärztenetzwerk die Zuwendungen an die Netzärzte weitergibt oder den Netzärzten selbst Zuwendungen (z.B. Rabatte) gewährt, gilt das Ärztenetzwerk als Vorteilsgeber im Sinne von Art. 33 HMG und die entsprechenden Vorschriften sind zu beachten.

Im einzelnen gilt folgendes (vgl. auch [1, 2]):

- Rabatte, die ein Ärztenetzwerk von seinem Lieferanten erhält, müssen nicht an die Versicherten des Ärztenetzwerkes weitergegeben werden. Das Ärztenetzwerk kann die Rabatte patientenbezogen oder für Projekte einsetzen. Voraussetzung ist, dass das Verschreibungs- und Abgabeverhalten der Netzärzte nicht beeinflusst wird.

- Rabatte, die ein Ärztenetzwerk von seinem Lieferanten erhält und an die Netzärzte weiterleitet, müssen von den Netzärzten bei der Einzelleistungsvergütung an die Patienten weitergegeben oder - im Falle von Pauschalen für die Versicherten des Ärztenetzwerkes - in den Verhandlungen mit den Krankenversicherungen berücksichtigt werden.

- Rabatte, die ein Ärztenetzwerk den einzelnen Netzärzten gewährt, müssen handelsüblich oder betriebswirtschaftlich gerechtfertigt sein. Handelsübliche Rabatte müssen 
von den Netzärzten an die Patienten oder die Krankenversicherungen weitergegeben werden. Betriebswirtschaftlich gerechtfertigte Rabatte müssen von den Netzärzten nicht weitergegeben werden, falls diese eine handelsbezogene Leistung erbringen, die üblicherweise entschädigt wird (z.B. Mengenrabatt bei Bestellung einer grösseren Menge). Rabatte, die höher sind als handelsüblich oder die nicht betriebswirtschaftlich gerechtfertigt sind, sind nicht zulässig.

- Sponsoringbeiträge, die ein Ärztenetzwerk von Dritten erhält, sind für diejenigen Zwecke einzusetzen, für die sie bestimmt sind und gewährt werden. Sie müssen nicht weitergegeben werden. Voraussetzung ist jedoch, dass das Gewähren des Sponsoringbeitrags keinen Einfluss hat auf das Verschreibungs- und Abgabeverhalten der Netzärzte und nicht der Umgehung von gesetzlichen Vorschriften zum Umgang mit Vergünstigungen dient.

- Finanzielle Beiträge von Dritten an ein Ärztenetzwerk, die betragsmässig über handelsübliche und/oder betriebswirtschaftlich gerechtfertigte Rabatte hinausgehen oder nicht patientenbezogen oder für ein Netzwerkprojekt resp. eine Netzwerkaktivität eingesetzt werden, sind nicht zulässig, falls sie Einfluss haben auf das Verschreibungs- oder Abgabeverhalten der Netzärzte.

- Damit die Zulässigkeit von Zuwendungen und die Weitergabepflicht beurteilt und letztere gegebenenfalls erfüllt werden kann, führen die Lieferanten Rabatte auf allen Rechnungen transparent und detailliert auf. Es empfiehlt sich, Sponsoring vertraglich zu regeln und die Projekte, für welche die Sponsoringbeiträge eingesetzt werden sollen, genau zu bezeichnen.

\section{Hinweise}

Solange es keine gefestigte Praxis der Behörden oder gerichtlichen Entscheide gibt, ist die Zulässigkeit von Rabatten und Sponsoringbeiträgen sowie die Pflicht zu ihrer Weitergabe im Einzelfall zu prüfen. Massgebend ist insbesondere, ob Leistung und Gegenleistung marktgerechten Bedingungen entsprechen und ein genügender Zusammenhang zwischen dem geldwerten Vorteil und dem konkreten Verschreibungs- oder Abgabeverhalten der Netzärzte besteht.

Bei Zuwendungen an Ärztenetzwerke, die im Ärztenetzwerk selbst verwendet werden, kann in der Regel davon ausgegangen werden, dass der Zusammenhang zwischen der Zuwendung und dem Verschreibungs- oder Abgabeverhalten der
Netzärzte genügend abgeschwächt wird und Art. 33 HMG nicht greift [3]. Falls die Netzärzte jedoch zum Beispiel verpflichtet werden, die (günstig) eingekauften Arzneimittel abzugeben, statt für einen bestimmten Patienten besser geeignete Arzneimittel zu verschreiben, hat die Vergünstigung mit hoher Wahrscheinlichkeit Einfluss auf das Abgabeverhalten der Netzärzte. Solche Situationen stehen im Widerspruch zu Art. 33 HMG und sind zu vermeiden. Das gleiche gilt auch für Exklusivverpflichtungen.

Schliesslich ist darauf hinzuweisen, dass verschiedene Kantone Regelungen kennen über die Zusammenarbeit zwischen verschiedenen Medizinalpersonen oder von Medizinalpersonen mit Dritten sowie für die Berufsausübung. Diese sind gegebenenfalls auch zu beachten.

\subsection{Beispiel: Einkauf und Verkauf von Impfstoff mit Rabatt}

\begin{tabular}{ll} 
Publikumspreis & Fr. 8.- \\
\hline betriebswirtschaftlich gerechtfertigter Rabatt & Fr. 1.- \\
\hline handelsüblicher Rabatt & Fr. 2.- \\
\hline Einkaufspreis für Ärztenetzwerk & Fr. 5.-
\end{tabular}

- Das Ärztenetzwerk muss weder den handelsüblichen noch den betriebswirtschaftlich gerechtfertigten Rabatt (Fr. 2.- und Fr. 1.- pro Impfstoff) weitergeben, sondern kann beide für patienten- oder netzwerkbezogene Projekte einsetzen.

- Gibt das Ärztenetzwerk den handelsüblichen und/oder den betriebswirtschaftlich gerechtfertigten Rabatt, den es selbst erhalten hat, an die Netzärzte weiter, gelten diese Rabatte für die Netzärzte als Vergünstigung ohne entsprechende Gegenleistung und sind in diesem Umfang (Fr. 2.- und/oder Fr. 1.-) an die Patienten oder deren Krankenversicherung weiterzugeben. Falls jedoch ein Netzarzt selbst eine handelsbezogene Leistung gegenüber dem Ärztenetzwerk erbringt, die üblicherweise entschädigt wird, muss er diese Entschädigung nicht weitergeben (z.B. wenn er für den Einkauf einer grösseren Menge einen Mengenrabatt erhält).

- Gibt das Ärztenetzwerk den Impfstoff unentgeltlich an die Netzärzte weiter (z.B. für eine Impfkampagne), erhalten die Netzärzte ebenfalls eine Vergünstigung ohne entsprechende Gegenleistung. Die Vergünstigung ist in vollem Umfang an die Patienten oder deren Krankenversicherung weiterzugeben (beispielsweise, indem der Impfstoff unentgeltlich abgeben wird). 


\section{Gesetzliche Grundlagen für den Umgang mit Zuwendungen}

Massgebend sind Art. 33 HMG (SR 812.21) und Art. 56 Abs. 3 KVG (SR 832.10).

\subsection{Wortlaut von Art. 33 HMG: Versprechen und Annehmen geldwerter Vorteile}

1 Personen, die Arzneimittel verschreiben oder abgeben, und Organisationen, die solche Personen beschäftigen, dürfen für die Verschreibung oder die Abgabe eines Arzneimittels geldwerte Vorteile weder gewährt noch angeboten noch versprochen werden.

2 Personen, die Arzneimittel verschreiben oder abgeben, und Organisationen, die solche Personen beschäftigen, dürfen für die Verschreibung oder die Abgabe von Arzneimitteln geldwerte Vorteile weder fordern noch annehmen.

3 Zulässig sind jedoch:

a. geldwerte Vorteile von bescheidenem Wert, die für die medizinische oder pharmazeutische Praxis von Belang sind;

b. handelsübliche und betriebswirtschaftlich gerechtfertigte Rabatte, die sich direkt auf den Preis auswirken.

\subsection{Wortlaut von Art. 56 Abs. 3 KVG: Wirtschaftlichkeit der Leistungen}

[...]

1 Der Leistungserbringer muss dem Schuldner der Vergütung die direkten oder indirekten Vergünstigungen weitergeben, die ihm:

a. ein anderer in seinem Auftrag tätiger Leistungserbringer gewährt;

b. Personen oder Einrichtungen gewähren, welche Arzneimittel oder der Untersuchung oder Behandlung dienende Mittel oder Gegenstände liefern.

\subsection{Begriffe}

Rabatte und Sponsoringbeiträge gelten als geldwerte Vorteile oder Vergünstigungen. Zurzei gibt es noch keine einheitliche oder gefestigte Auslegung und keine Praxis der Behörden oder gerichtlichen Entscheide. Im folgenden wird deshalb die zurzeit vorherrschende Meinung zur Auslegung und Anwendung der erwähnten Begriffe zusammengefasst.

\section{Geldwerte Vorteile und Vergünstigungen}

Als geldwerte Vorteile oder Vergünstigungen gelten direkte finanzielle Vorteile sowie weitere Zuwendungen im Sinne einer wirtschaftlichen oder anderen materiellen Leistung. Der Vorteil bewirkt eine objektiv messbare Bes- serstellung, die ohne Gegenleistung oder ohne (überzeugenden) Rechtsgrund gewährt wird [4, 5].

Beispiele von geldwerten Vorteilen im $\mathrm{Zu}-$ sammenhang mit dem Heilmittelrecht sind Superboni, Reisen, Einladungen, Geschenke, Gratismuster [6], aber auch Bargeld, Gegenstände zur Nutzung oder Praxiseinrichtungen, etc. sowie das Bezahlen von überhöhten Beraterhonoraren [4]. Die Charta befasst sich nicht mit geldwerten Vorteilen, welche von Dritten direkt an einzelne Netzärzte gewährt werden, wie Reisen, Einladungen an Kongresse, Gratismuster, Geschenke usw. Für den Umgang mit solchen Zuwendungen wird auf die Empfehlungen resp. Richtlinien der Schweizerischen Akademie der Medizinischen Wissenschaften [7] oder den Verhaltenskodex der pharmazeutischen Industrie in der Schweiz [8] verwiesen.

\section{Handelsübliche Rabatte}

Handelsübliche Rabatte sind Vergünstigungen, welche im Handel bei bestimmten Produkten oder Produktgruppen im Einzelfall während längerer Zeit gewährt worden sind und betriebswirtschaftliche Rabatte übersteigen. Ihre Vorhersehbarkeit bewirkt, dass ein handelsüblicher Rabatt keinen Einfluss auf die Kaufentscheidung hat $[1,2,9]$.

\section{Betriebswirtschaftlich gerechtfertigte Rabatte} Betriebswirtschaftlich gerechtfertigten Rabatten stehen handelsbezogene, wirtschaftliche Leistungen des Empfängers gegenüber. Der Rabattempfänger übernimmt im Rahmen der Vertriebskette Aufgaben, welche sonst vom Produzenten oder Lieferanten wahrgenommen werden. Dazu gehören beispielsweise spezielle Zahlungskonditionen (Skonti bei frühzeitiger Bezahlung, vereinfachtes Bestellverfahren [Online-Bestellungen] usw.), die Abgeltung des reduzierten logistischen Aufwands beim Verkäufer (Grossmengenlieferung, reduzierte Lieferungshäufigkeit oder Lagerhaltung durch die Einkäuferin) oder die Abgeltung anderer Dienstleistungen des Einkäufers wie Datenaufbereitung oder Ablaufstandardisierung $[2,9]$.

\section{Sponsoringbeiträge}

Beim Sponsoring wird eine finanzielle oder geldwerte Unterstützung gewährt zur Förderung von Organisationen oder Personen in bestimmten Bereichen. Der Sponsor verfolgt dabei auch eigene, unternehmensbezogene Ziele. In der Regel verlangt er, dass die Tatsache seines Sponsorings bekanntgegeben wird, um beispielsweise seinen Bekanntheitsgrad zu steigern 
oder Imagewerbung zu betreiben. Beim Sponsoring werden die Mittel nicht voraussetzungslos gewährt, sondern zweckbestimmt und nach dem Prinzip von Leistung und Gegenleistung $[10,11]$.

\subsection{Weitere Erläuterungen zu Art. 33 HMG und Art. 56 Abs. 3 KVG}

\section{Zusammenhang zwischen dem geldwerten Vorteil und dem Verschreiben und Abgeben von Arzneimitteln gemäss Art. 33 HMG}

Art. 33 HMG greift nur, wenn die geldwerten Vorteile «für» Verschreiben oder Abgeben von Arzneimitteln gewährt oder angenommen werden. Ob der verlangte Zusammenhang zwischen dem Gewähren eines Vorteils und dem Verschreiben und Abgeben von Arzneimitteln im Einzelfall besteht, kann schwierig zu bestimmen sein. So sind zum Beispiel zweckgebundene finanzielle Beiträge zur Unterstützung von Forschung und Entwicklung von Arzneimitteln auch zulässig an Personen, die Arzneimittel verschreiben oder abgeben sowie an Organisationen, die solche Personen beschäftigen. Werden solche Beiträge jedoch im Hinblick auf das Bestellen von Arzneimitteln oder als Belohnung für «gute Verschreiber» gewährt, sind sie nicht zulässig.

Damit Art. 33 HMG anwendbar ist, muss ein hinreichend enger Zusammenhang bestehen zwischen dem Vorteil und dem Verschreibungsund Abgabeverhalten. In der Praxis wird zur Beurteilung auf objektive Momente wie die Höhe der Zahlungen, die zeitliche Nähe von Leistung und Gegenleistung oder die Häufigkeit der Kontakte abgestellt [4].

\section{Preisauswirkungsgebot nach Art. 33 HMG}

Art. 33 HMG verlangt, dass sich Rabatte auf den Preis auswirken. Die Bedeutung der Wendung «[...] die sich direkt auf den Preis auswirken» am Schluss in Art. 33, Abs. 3, lit. b, HMG ist noch nicht geklärt. Die Auslegung, wonach diese Vorschrift eine Weitergabe der Rabatte an Patienten oder Krankenversicherer verlangt [1], steht im Widerspruch zum klaren Wortlaut der Bestimmung und - da die Weitergabe von Vergünstigungen als sozialpolitisch motivierte Pflicht betrachtet wird - im Widerspruch zum Zweck des Heilmittelgesetzes und Art. 33 HMG. Einleuchtender scheint die Auslegung, dass Rabatte in Form von Preisnachlässen zu gewähren sind und sich damit direkt «auf den Preis auswirken», der vom Empfänger zu bezahlen ist. In diesem Sinne sind Rabatte unzulässig, welche nicht in Form von direkten Preisnachlässen, sondern z.B. als Rückvergütung, Pauschale oder Koppelungsgeschäft gewährt werden $[2,3]$.

\section{Unterschiede zwischen Art. 33 HMG und Art. 56}

Abs. 3 KVG

Sowohl Art. 33 HMG als auch Art. 56 Abs. 3 KVG befassen sich mit Vergünstigungen, sie unterscheiden sich aber im Zweck und teilweise im Anwendungsbereich:

- Art. 33 HMG soll im Zusammenhang mit Art. 26 HMG sicherstellen, dass der Entscheid über das im Einzelfall verschriebene oder abgegebene Arzneimittel nur medizinischen oder pharmazeutischen Kriterien folgt und nicht von finanziellen Anreizen beeinflusst wird. Das Krankenversicherungsgesetz demgegenüber hat einen sozialpolitischen Zweck und soll die Wirtschaftlichkeit der vergüteten Leistungen sicherstellen.

- Art. 33 HMG gilt für alle Arzneimittel (SL- und Nicht-SL-Produkte), nicht aber für Medizinprodukte. Art. 56, Abs. 3, KVG gilt nur für die Arzneimittel aus der SL und die Medizinprodukte aus der MiGeL.

- Art. 33 HMG verbietet geldwerte Vorteile mit Ausnahme von bestimmten - umfangmässig beschränkten - Ausnahmen. Art. 56, Abs. 3, KVG demgegenüber verbietet Vergünstigungen nicht, sondern verlangt, dass diese dem Patienten oder seiner Krankenversicherung weitergegeben werden.

- Art. 33 HMG greift nur, wenn ein Zusammenhang besteht mit Verschreiben oder Abgeben von Arzneimitteln. Dieser Zusammenhang muss einigermassen deutlich sein, und er muss vor allem geeignet sein, das Verschreibungs- oder Abgabeverhalten der Medizinalpersonen zu beeinflussen [1, 9]. Art. 56, Abs. 3, KVG verlangt keinen Zusammenhang mit Verschreiben oder Abgeben.

Wieweit sich diese Unterschiede in der Praxis auswirken, ist zurzeit noch nicht geklärt.

\section{Literatur}

1 Swissmedic. Zulässigkeit von Rabatten im Rahmen von Artikel 33 Absatz 3 Buchstabe b des Heilmittelgesetzes. Swissmedic Journal 2003;11:980.

2 Empfehlung des BSV betreffend die Weitergabe der beim Einkauf verwendungsfertiger Arzneimittel erhaltenen Vergünstigungen im ambulanten Bereich vom 11. Juli 2002.

3 Saxer U. Das Vorteilsverbot gemäss Art. 33 HMG Erste Erfahrungen und Folgen. In: Eichenberger T, Poledna T (Hrsg.). Das neue Heilmittelgesetz. Zürich: Schulthess; 2004. 133 ff.

4 Botschaft zur Revision des Korruptionsstrafrechts. BBl 19995527. 
5 Baudenbacher C. Lauterkeitsrecht. Basel: Helbing \& Lichtenhahn; 2001. Rz. 45 f. zu Art. 4 UWG.

6 Botschaft zum Heilmittelgesetz. BBl 1999 3453, 3518.

7 SAMW. Zusammenarbeit Ärzteschaft - Industrie. Empfehlungen der Schweizerischen Akademie der Medizinischen Wissenschaften vom 9. September 2002. Schweiz Ärztezeitung 2002;83(41):2165-1; überarbeitet und als Richtlinien in die Vernehmlassung gegeben im Januar 2005. Schweiz Ärztezeitung 2005;86(2):108-14.

8 Verhaltenskodex der pharmazeutischen Industrie in der Schweiz (Pharmakodex) vom 4. Dezember 2003; abrufbar unter www.sgci.ch.
9 Gutachten der Wettbewerbskommission vom 1. September 2003 zuhanden der Preisüberwachung über die Auslegung von Artikel 33 HMG, RPW 2003;3:623.

10 Arzethauser M. Die Vorteilsgewährung bzw. die Vorteilsannahme nach dem revidierten Schweizerischen Korruptionsstrafrecht. Rapperswil: Berti Druck; 2001.

11 Hauser T. Der Sponsoring-Vertrag im schweizerischen Recht. Zürich: Schulthess; 1991. 